Research Article

\title{
The Diagnostic Value of Cystatin C and Mild Hypothermia Therapy Based on Immunoturbidimetry Enhanced by Nanospheres in Asphyxia Neonate
}

\author{
Xiaoping Dang, ${ }^{1}$ Xiaojian Hu $\mathbb{D}^{2}{ }^{2}$ Yuancui Meng, ${ }^{1}$ Yan'e Yang, ${ }^{3}$ Lingfang Zheng, \\ Qiong Zhang, ${ }^{4}$ Nan Dang, ${ }^{5}$ Jing Hao, ${ }^{6}$ Liang Zheng, ${ }^{2}$ and Feng $\mathrm{Ni}^{2}$ \\ ${ }^{1}$ Department of Neonatal, Second Affiliated Hospital of Xi'an Medical College, Xi'an 710038, China \\ ${ }^{2}$ Department of Urology, Second Affiliated Hospital of Xi'an Medical College, Xi'an 710038, China \\ ${ }^{3}$ Department of Pediatrics, Second Affiliated Hospital of Xi'an Medical College, Xi'an 710038, China \\ ${ }^{4}$ Department of Nephrology, Second Affiliated Hospital of Xi'an Medical College, Xi'an 710038, China \\ ${ }^{5}$ Department of Pharmacy, Second Affiliated Hospital of Xi'an Medical College, Xi'an 710038, China \\ ${ }^{6}$ Department of Neonatology, Affiliated Hospital of Yan'an University, Yan'an, China \\ Correspondence should be addressed to Xiaojian Hu; 19084943@qq.com
}

Received 24 July 2020; Revised 27 October 2020; Accepted 15 November 2020; Published 12 December 2020

Academic Editor: Tifeng Jiao

Copyright ( 92020 Xiaoping Dang et al. This is an open access article distributed under the Creative Commons Attribution License, which permits unrestricted use, distribution, and reproduction in any medium, provided the original work is properly cited.

\begin{abstract}
In order to evaluate the early diagnosis value of $\mathrm{CysC}$ and the influence of mild hypothermia on the renal damage of asphyxia neonates, the serum cystatin $\mathrm{C}(\mathrm{CysC})$ levels of asphyxia neonates and normal neonates were measured by the nanomicrosphereenhanced immunoturbidimetric method. The treatment was carried out, and the influence of mild hypothermia treatment on the renal damage of asphyxia neonates was analyzed. The results showed that the indicators of the asphyxia group were significantly higher than those of the control group, and the severe asphyxia group was significantly higher than that of the mild asphyxia group, which was statistically significant $(p<0.05)$; the heart rate of patients in the mild hypothermia treatment group decreased gradually with the decrease in body temperature. And compared with the control group, there was a significant difference $(p<0.05)$; after symptomatic treatment, the two groups of ALT, AST, BUN, and SCR were improved to varying degrees, and the difference was statistically significant compared with before treatment $(p<0.05)$. Studies have shown that serum CysC level can be used as an indicator to detect glomerular filtration function and early asphyxia newborns, and it is sensitive and specific for early diagnosis of kidney damage. At the same time, it can be used to monitor clinical renal function and determine the status of asphyxia newborns.
\end{abstract}

\section{Research Background}

Neonatal asphyxia easily leads to multiple organ ischemia, hypoxia, and functional damage; therefore, early protection of renal function is of great significance [1]. According to a longterm follow-up study, if kidney damage occurs in the neonatal period, neonatal asphyxia is caused by various reasons before, during, and after birth [2]. It is one of the important causes of perinatal neonatal death and disability [3].

In China, the incidence of asphyxia is higher than 5\%, and asphyxia death accounts for more than $30 \%$ of infant death [4]. The main complications of asphyxia are dysfunction of brain, kidney, heart, and other organs, and kidney is one of the most vulnerable target organs $[5,6]$. Neonatal asphyxia often occurs and is even life-threatening in severe cases. When asphyxia occurs, the endogenous creatinine clearance rate will decrease, nitric oxide in the kidney will increase, and the synthesis and expression of adhesion molecules between cells will increase, which can easily lead to impaired renal function [7]. Renal function usually includes glomerular filtration and reabsorption and secretion of renal tubules. 
Glomerular filtration rate (GFR) is the most direct, objective, and sensitive indicator of glomerular filtration rate. It cannot be measured directly, but it can be measured by measuring the filter rate of a specific marker. For example, inulin removal and isotope labeling are external labels and the gold standard for evaluating glomerular filtration rate $[8,9]$. However, this kind of special operation is complex, expensive, and invasive, which is difficult to promote in newborns. There are three forms of creatinine (CR): serum creatinine clearance rate, endogenous creatinine clearance rate, and blood creatinine glomerular filtration rate. These indexes are affected by many factors and are easily disturbed [10]. Although it is reabsorbed by the epithelial cells of the proximal convoluted tubules, it then decomposes in the cells, so the glomerular YSC does not return to the blood $[11,12]$. From these characteristics, cystatin $\mathrm{C}$ is close to the ideal endogenous GFR marker reflecting the early damage of renal filtration barrier. The serum level is relatively stable and free from the interference of bilirubin, hemoglobin, cholesterol, age, gender, exercise, diet, inflammation, and other external factors [13]. It is more accurate and sensitive than SCR. Early diagnosis and treatment of renal damage are necessary to reduce mortality and improve the poor prognosis of renal damage.

\section{Related Content}

2.1. Neonatal Asphyxia. Various high-risk factors before, during, and after childbirth cause fetal distress, lead to anoxia and acidosis, and then damage the multiorgan function of the whole body. Immature renal function, poor glomerular filtration, poor reabsorption and excretion of renal tubules, asphyxia, and hypoxia stimulate arachidonic acid metabolism, increase the synthesis of thromboxane A2, make renal vessels contract, reduce renal blood flow, and reduce glomerular filtration rate $[14,15]$. Severe hypoxia can cause renal cortex and medulla necrosis, nephron damage, abnormal cell energy metabolism, lactic acid accumulation, acidosis damage cell membrane pump function, and calcium influx. After asphyxia, proximal convoluted tubules and glomerular filtration membranes are sensitive to hypoxia and ischemia. The renal hemodynamics, renal blood flow velocity, and systolic blood flow velocity of asphyxiated neonates were monitored by color Doppler technique. The physiological characteristics of newborns and the pathophysiological changes after renal hypoxia can lead to acute renal failure, such as functional oliguria, anuria, hematuria, albuminuria, tubular urine, water, electrolyte, acid-base imbalance, etc. [16].

The incidence rate of neonatal is not low, but if the early intervention is reversible and the condition is aggravated, renal failure may occur, and life is seriously endangered. In addition, when neonatal renal function is damaged, the symptoms are not typical and early complications are difficult to distinguish. Therefore, early diagnosis is very important for the prevention and treatment of acute renal failure. Renal function includes glomerular filtration function, renal tubular reabsorption function, and secretion function [17]. Glomerular filtration rate is the most direct, objective, and sensitive index to reflect glomerular filtration function. With the development of medicine and the improvement of renal function monitoring methods, more and more reports on renal function damage of newborn are presented. Neonatal asphyxia is one of the most common and important diseases endangering the health of newborn [18]. Metabolic or mixed acidosis caused by hypoxia and inhibition of central nervous system and respiratory circulation often develops into multiple organ damage, in which the incidence of renal damage is more than $50 \%$. Therefore, the application of sensitive renal function detection method in the monitoring of renal damage after asphyxia is of positive significance in guiding clinical treatment and reducing the mortality and disability rate of asphyxiated newborns. In the past, serum creatinine and urea nitrogen were commonly used to monitor renal function, but the serum level of these markers was not stable, generally only increased when the glomerular filtration function lost more than $50 \%$. Therefore, serum creatinine and urea nitrogen should not be used as indicators to detect mild or early glomerular filtration damage. There are also some low molecular weight proteins, such as microglobulin, and retinol binding protein. Microglobulin has been proved to reflect the change of glomerular filtration rate, but its level in blood circulation will be affected by infection, diet, liver disease, and other external factors, so as to change its production rate and limit its clinical application. The analysis of its gene sequence and promoter structure shows that cystatin C gene is a "housekeeping gene," which can be continuously transcribed and expressed in all tissues $[19,20]$. All nucleated cells can secrete cystatin C.

2.2. Early Diagnosis Index. Routine urine examination often shows renal function damage: urine contains red blood cells, white blood cells, protein, renal tubular epithelial cells, and renal tubular type, which is the earliest and most common urinary system examination method with high sensitivity. Tongjianxia reported that it has clinical significance for asphyxiated newborns, but it is affected by collection time, placement time, urine chemical composition, and other factors, and the difficulty of urine collection for newborns is great, so its application value in neonatal asphyxia is limited. It is affected by protein intake, decomposition level, renal blood flow, and other factors. Only when the glomerular filtration rate drops to $1 / 3$ of the normal value can the creatinine increase significantly [21, 22]. The creatinine level of the newborn represents the level of the pregnant woman, which is usually high. With the maturity of renal function, the clearance rate increased and SCR decreased. Therefore, detection is limited and can not predict early renal function damage. When renal function decreased, the increase of blood BUN and Cr was not directly related to the decrease of glomerular filtration rate, but in the form of antiparabola. That is, in asphyxia and hypoxia, glomerular filtration rate decreased and serum creatinine increased. Renal compensatory function is powerful; only when more than $75 \%$ of nephrons are destroyed and the glomerular filtration rate is reduced to less than $50 \%-60 \%$, it can increase the blood 
capsule and blood $\mathrm{Cr}$, which has a more obvious reflection, and the sensitivity of the test is poor, so it is possible to miss the early pathological changes or slight renal damage of patients. Therefore, diagnosis may be delayed, which is not an indicator of early renal impairment. At present, the detection of urea nitrogen and creatinine is easy to carry out, and its practical value is still a routine item for the diagnosis of neonatal renal function in primary hospitals. Endogenous creatinine clearance rate (CCR) is a quantitative method which can reflect glomerular filtration function, roughly estimate effective renal units, and measure renal damage. When CCR is lower than $80 \%$, blood BUN and SCR can still be in the normal range and CCR can reflect the impairment of glomerular function earlier. It is a suitable diagnostic method for the neonates with normal urine volume and azotemia. However, CCR is calculated based on the concentration of creatinine in the blood and urine. It still has the disadvantages of SCR. It is also influenced by many factors. For example, if you want to take a total urine volume of 4 or 24 hours, the retention sample may not be accurate and the measurement process is complex. In order to clear the plasma, the ML value of creatinine per minute must be calculated. It is difficult and limited for newborns. More than 99.9\% of them enter the cytoplasm through the pinocytosis of renal proximal convoluted tubules and decompose into amino acids, so only a very small part of them are excreted from urine. Therefore, under normal circumstances, there is only a very small amount of microglobulin in human urine, so the microglobulin in urine is an indicator of kidney damage. In patients with acute and chronic renal failure, urinary $\beta 2$-microglobin increased significantly, $40 \mathrm{mg} / \mathrm{L}$, which proved that such a high level of urinary $\beta 2$-microglobin could not be simply explained as the increase of glomerular filtration load, and renal tubules must also suffer serious damage. The increase of serum content can reflect the impairment of glomerular filtration function or the increase of filtration load. With the development of the disease and the increase of the age of the newborn, the improvement of the degree of hypoxic ischemia and the decrease of $\beta 2$-mg in serum are the sensitive indexes for the early recovery of renal tubular function.

It is of great clinical value to check the change of $\beta 2-\mathrm{mg}$ in blood and urine regularly for early detection of diabetic nephropathy. The detection of cystatin $\mathrm{C}$ (CysC) is an immunological method based on antigen antibody reaction. The sensitivity of enzyme-linked immunosorbent assay is high, and the accuracy of detection is not affected by bilirubin, hemoglobin, and other factors in the blood. Its accessory characteristics meet the requirements of ideal endogenous GFR markers: glomerular free filtration; no secretion or reabsorption of renal tubules; and no external space; it is stable in vivo, appears in plasma, and does not bind with protein; it is easy to detect in plasma and urine. More and more attention has been paid to the study of serum cystatin C. Fang Mingjun and QiuYuanyuan reported that the more severe the asphyxia, the higher the level of cystatin C. The serum concentration of premature infants was higher than that of term infants. In the two stages of preterm and full-term infants, it was not affected by inflammation, bilirubin level, hemoglobin content, hemolytic factor, triacylglycerol, weight, and liver function change, and had nothing to do with gender, age, muscle volume, etc. The high concentration of $\mathrm{sCysC}$ in the early stage of life is related to the maturation of glomerular filtration function. With the gradual maturity of glomerular filtration function, the concentration tends to be constant. The formation of cystatin $\mathrm{C}$ in neonates is not affected by maternal factors or prerenal factors (i.e., dehydration). Its metabolism is only affected by glomerular filtration rate. It mainly exists in extracellular fluid, but there is almost no such substance in urine; that is, the level of cystatin $\mathrm{C}$ in urine is very low, so it is not necessary to evaluate renal function through cystatin clearance rate. So, the concentration of CysC in blood can be used as a sensitive index to reflect the early damage of glomerular filtration barrier and can better reflect the change of glomerular filtration rate, which is better than creatinine and urea nitrogen in the early stage of renal failure. We know that $\mathrm{CysC}$ is completely absorbed and metabolized in proximal renal tubules after glomerular filtration. The urine content of normal people is very small. Therefore, in theory, when the renal tubular reabsorption or/and metabolic function is damaged, the concentration of urinary $\mathrm{CysC}$ ( $\mathrm{uCysC}$ ) can be greatly increased. It is speculated that $\mathrm{uCysC}$ can reflect the function of proximal renal tubular. Some studies have shown that the increase of uCysC can not accurately reflect the damage of GFR, but it is very sensitive to reflect the damage of renal tubular function. Compared with the abovementioned traditional detection, sCysC is theoretically more sensitive than $\mathrm{sCysC}$ in reflecting GFR, especially in the case of a slight decrease in GFR, because of its unique molecular biological and biochemical characteristics. Compared with SCR, sCysC has higher diagnostic accuracy and sensitivity. SCR was not reported to be superior to sCysC. sCysC is superior to SCR in monitoring acute renal injury in critical children. $\mathrm{sCysC}$ is a simple and useful GFR marker, which is of great value for monitoring renal function in critically ill children. $\beta 2-\mathrm{mg}$ is also a marker of GFR, but it is affected by many factors such as inflammation and tumor. The incidence of renal function damage after neonatal asphyxia is in brain injury. The detection of blood BUN and SCR is of practical value in primary hospitals and is still the most commonly used indicator for diagnosis and monitoring. The prognosis of early diagnosis and treatment is good. It is difficult to find the early clinical symptoms of neonatal renal damage, so it is necessary to monitor renal function with a variety of sensitive indicators. In conclusion, $\mathrm{CysC}$, as a new early indicator of renal function damage, has been evaluated as more sensitive or equivalent to SCR in many pediatric clinical research fields. Whether we can replace SCR as an indicator of renal function damage in clinical work remains to be further designed and scientifically studied to evaluate its clinical applicability.

2.3. Immunoturbidimetry Enhanced by Nanospheres. Polymer microsphere is a kind of composite material or polymer material whose diameter is between micrometer and 
nanometer and whose shape is generally spherical. There are many preparation methods in the literature. At present, there are two main categories of polymers, including emulsion curing, single coacervation, and complex coacervation. The other is polymerization with monomer as raw material. According to the properties of microspheres, they can be divided into magnetic microspheres, fluorescent microspheres, and so on. According to the different surface functional groups, it can be divided into carboxylation, amination, sulfonation polymer microspheres, and so on. With the introduction of various functional groups into monodisperse polymer microspheres, their applications are more and more extensive. At present, it is mainly used in biomedical, analytical chemistry, colloid, in vivo drug analysis, and chromatographic separation. It shows a good application prospect in the field of high and new technology. At present, polymer microspheres have been commercialized in Europe, the United States, Japan, and other regions. Styrene is often used as the raw material monomer for the preparation of polymer microspheres because of its low price, easy access to raw materials, and easy polymerization. In order to detect and diagnose diseases, latex enhanced immunoturbidimetry (letia) is often used. By measuring the change of a value before and after the reaction, the concentration of antibody (antigen) in the sample can be calculated. Letia has been widely used in clinical, involving tumor, rheumatism, liver function, renal function, and other fields. Emulsion polymerization is the most commonly used method to prepare microspheres. The surface of PS microspheres is phenyl with strong hydrophobicity and low reactivity. It is not easy to react with antibodies, antigens, and other proteins. The antibody or antigen is physically adsorbed on the surface of the microspheres through the hydrophobic interaction between the hydrophobic part and phenyl. The physical adsorption is nonspecific and unstable. The adsorption of antibody on the surface of the microspheres will decrease with the prolongation of the reagent storage time. At the same time, when the microsphere adsorption containing solution contacts with other protein antibodies, the adsorbed antibodies are exchanged with the protein solution, so that the microsphere loses detection activity. Therefore, the stability and sensitivity of diagnostic reagents are affected. If carboxy or amino groups react strongly to introduce microspheres to immobilize antibody and microspheres through chemical bonding reaction, the firmness of antibody binding and microspheres can be enhanced to a large extent, and the phenomenon of antibody shedding and protein exchange can be avoided. The common methods of introducing active groups are copolymerization of different monomers and chemical modification of microsphere surface. Immunoassay is a method to detect various substances (drugs, proteins, microorganisms, etc.) by the specific binding reaction of antibody antigens.

At present, detection reagents are commonly used in clinical latex enhanced immunoassay, including $\beta 2$ microglobulin detection reagent $(\beta 2-\mathrm{m}), \mathrm{C}$ protein detection reagent, pulmonary embolism detection reagent, glycosylated hemoglobin detection reagent, etc., which can be, respectively, used in the detection and diagnosis of renal function, rheumatism, blood lipid thrombosis, diabetes, etc. The carriers commonly used in immunoassay include test tubes, microspheres, magnetic particles, nanospheres, and other materials. Because the molecular structure of polymer microspheres can be designed, more and more scientists are engaged in this field. In order to achieve the required specific properties and functions, the size and uniformity of microspheres can be controlled purposefully in the design and preparation process. The whole process is divided into growth period, constant speed period, and deceleration period. In the reaction process, the amount of initiator and emulsifier will affect the size and distribution of microspheres. In order to stabilize emulsion polymerization, the amount of emulsifier and initiator dosage should be appropriately prepared to obtain emulsion with uniform particle size. At the same time, in order to prevent the particles from polycondensation, it is beneficial to the stability of emulsion polymerization to reduce the reaction temperature and the stirring speed under the premise of ensuring the polymerization effect. In order to improve the probability of obtaining free radicals, lipophilic initiator is often used to initiate polymerization, which is a kind of "bulk polymerization" of single drop of small body liquid. Initiator and other factors affect the preparation of suspension polymer microspheres. With the increase of the initial dose, the particle size increases and the polymerization time shortens. The adsorption rate of stabilizer is relatively low, which can accelerate the agglomeration process and easily produce larger beads. If the amount of dispersant is too high and the viscosity of the system is too high, the amount of nucleation will be increased. The growth of polymer microspheres was affected by the inhibition of nuclear polymerization. The immobilized enzyme is practical and convenient for industrialization. The polymer microsphere with carboxyl functional group on the characteristic surface used in the test is to repair the surface of antigen monodisperse microsphere with immune cell substance of $-\mathrm{NH}_{2}$ under mild conditions, then increase the antibody, use the principle and fixed antigen antibody to make the microsphere agglomerate, and then use the spectrophotometer to determine whether agglomerate is to determine the number of immune cells. The principle of virus removal is similar to the purification and separation of biomacromolecules. In short, the nanomicrosphere-enhanced immunoturbidimetric method has the advantages of high throughput, easy automation, being simple and fast, no radioactive contamination, and suitability for the detection of large quantities of specimens. It is of great help to the diagnosis and treatment of newborns and improving the survival rate of newborns.

\section{Experimental Setup}

3.1. Research Object. The subjects of the study were 50 asphyxiated neonates with term asphyxia in our hospital. The gestational age was 30-45 weeks, and the birth weight was 2 . $5-3.0 \mathrm{~kg}$. According to the diagnosis and grading criteria of neonatal asphyxia in the fourth edition of Practical 
Neonatology, they were divided into mild and severe near asphyxia groups: 10 scores as Apgar score of $0-3$ in severe asphyxia group and 10 scores as Apgar score of 4-7 in mild asphyxia group. In the control group, 10 full-term healthy newborns were selected as the control group. There was no significant difference in sex, gestational age, birth weight, and age among the three groups.

3.2. Specimen Collection. On the first day, the third day, and the seventh day of asphyxia, $5 \mathrm{ml}$ of peripheral venous blood was taken from the patients of mild asphyxia group and severe asphyxia group, respectively, and serum was separated. In the control group, $5 \mathrm{ml}$ of peripheral venous blood was taken on the second day of birth. The levels of CysC, $\mathrm{BUN}, \mathrm{Cr}$, and $\beta 2$-mg were determined by ELISA.

3.3. Experimental Plan. Detection of cystatin C in serum: cystatin $\mathrm{C}$ is detected using an immunoassay. Absorption of reactive solutions at specific wavelengths has been measured. There is a positive correlation between turbidity and serum cystatin concentration.

Particle enhanced immunoturbidimetry (PET) principle: the diameter of particle, which is covalently bound by carbodiimide reaction, and the size of ciggintex particle, which is bound with antigen, are used to precipitate PEG6000 and turbidity, which are calculated by the turbidimetry method of 360 nautical miles, while a series of standards are the same. Operation: take serum or standard series and put into the reaction cup, add $10 \mathrm{gl}$ distilled water Shen sampling needle, add 23091 reaction buffer, mix well, add cysteine protease inhibitor $\mathrm{C}$ immune particle suspension and distilled water Shen reagent needle, and measure the absorbance in 240s. Subtract the blank reading from the standard tube absorbance and make a picture. Subtract the blank reading from the sample tube absorbance and check the concentration of cystatin C from the standard curve.

Determination of serum creatinine: automatic biochemical instrument was used. Urea test: use urea test kit to test.

Determination of $\beta 2$-mg: the content of $\beta 2-\mathrm{mg}$ in serum samples of three groups of patients was detected by automatic biochemical instrument.

Glomerular filtration rate (EGFR): $\mathrm{EGFR}=k *$ height $(\mathrm{CM}) / \mathrm{SCR}(\mathrm{mg} / \mathrm{D} 1)$, where $k$ is a constant, indicating the output of urine creatinine per unit lean body weight, 0.45 for term infants and 0.33 for low birth weight infants, which can accurately estimate glomerular filtration rate.

Statistical processing: SPSS17.0 statistical software package was used for statistical analysis. Before statistical comparison of continuous data, normal test and Levene test were used. The measurement data were expressed by means of mean and standard deviation, and the group $t$-test was used for comparison between groups. The counting data is expressed in percentage, and the comparison between groups is performed by chi square test or Fisher exact probability method. Pearson correlation analysis was used to analyze the correlation between serum CysC and $\beta 2$-mg and renal function indexes. The difference was statistically significant $(p=0.05)$. In order to evaluate the feasibility of joint detection, sensitivity, specificity, positive predictive value, negative predictive value, positive likelihood ratio, and negative likelihood ratio need to be calculated, respectively.

\section{Experimental Results}

\subsection{Cystatin C Comparison}

4.1.1. Laboratory Indicators. According to the statistical analysis of data, as shown in Figure 1 and Table 1, CysC, $\beta 2$ $\mathrm{mg}, \mathrm{SCR}$, and BUN in the asphyxia group were significantly higher than those in the control group, and those in the severe asphyxia group were significantly higher than those in the mild asphyxia group $(p<0.05)$. The levels of serum SCR, BUN, $\beta 2-\mathrm{mg}$, and CysC in asphyxia group decreased gradually. The indexes of the first day of life in the mild asphyxia group were higher than those in the control group, the difference was statistically significant $(p<0.05)$, but there was no statistical difference between the indexes of the third day and the seventh day of life in the mild asphyxia group and the control group. The indexes of the first day and the third day in the severe asphyxia group were higher than those in the control group and the mild asphyxia group $(p<0.05)$.

4.1.2. Diagnostic Value. According to the statistical analysis of data, as shown in Figure 2 and Table 2, EGFR was used as the evaluation standard of renal function damage in asphyxia group. Compared with the levels of CysC, $\beta 2-\mathrm{mg}$, SCR, and BUN on the first day after admission, the sensitivity and specificity of serum CysC were the highest. The difference was statistically significant $(p<0.05)$. There was no significant difference between the two groups $(p<0.05)$.

\subsection{Mild Hypothermia Treatment}

4.2.1. Changes in Vital Signs. According to the statistical analysis of data, as shown in Figure 3 and Table 3, the heart rate of patients in the mild hypothermia treatment group gradually decreased with the decrease of body temperature, and the central heart rate in the course of mild hypothermia treatment was significantly different from that before and in the control group $(p<0.05)$. After mild hypothermia treatment, heart rate recovered with body temperature. After 12 hours of mild hypothermia treatment, the heart rate of children in the mild hypothermia treatment group recovered completely, and there was no significant difference between the two groups. Mild hypothermia was used to treat no arrhythmia and severe bradycardia. There was no statistical difference between the treatment group and the control group. There was no significant difference in blood pressure between the two groups before and after treatment. There was no significant change in blood pressure in the mild hypothermia group. After mild hypothermia treatment, blood pressure slightly decreased with the rise of body 


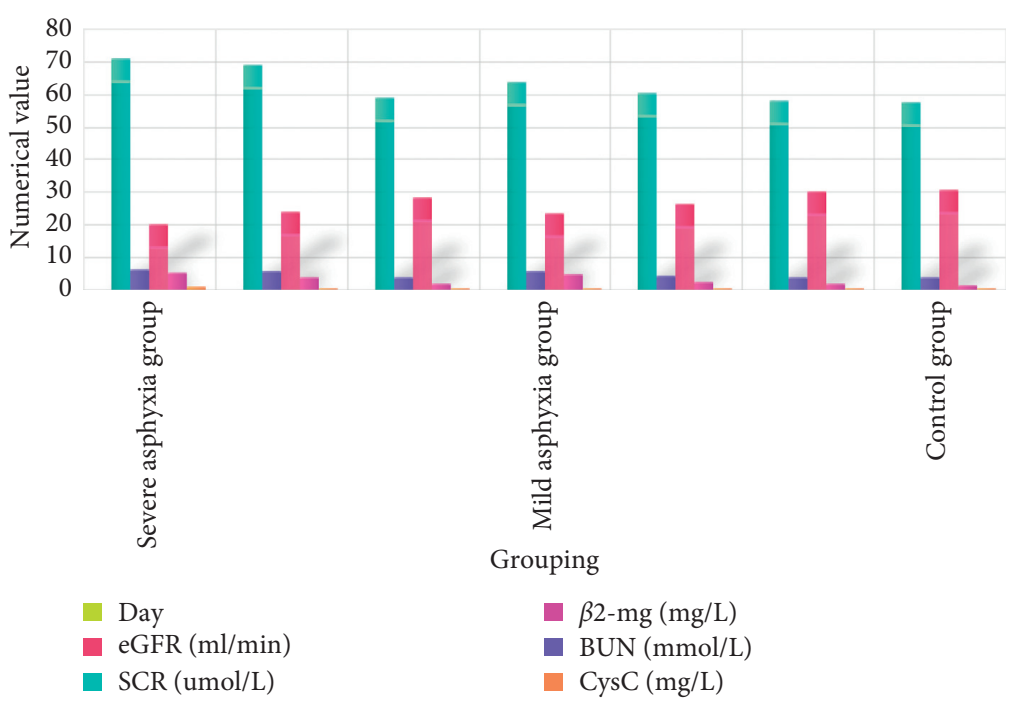

Figure 1: Laboratory indicators.

TABLE 1: Laboratory indicators.

\begin{tabular}{lcccccc}
\hline Grouping & & SCR $(\mu \mathrm{mol} / \mathrm{L})$ & BUN $(\mathrm{mmol} / \mathrm{L})$ & eGFR $(\mathrm{ml} / \mathrm{min})$ & $\beta 2-\mathrm{mg}(\mathrm{mg} / \mathrm{L})$ & $\mathrm{CysC}(\mathrm{mg} / \mathrm{L})$ \\
\hline \multirow{3}{*}{ Severe asphyxia group } & Day 1 & $72.0 \pm 7.32$ & $7.13 \pm 0.66$ & $21.11 \pm 4.02$ & $6.08 \pm 0.77$ & $1.54 \pm 0.25$ \\
& Day 3 & $69.7 \pm 6.88$ & $6.48 \pm 0.51$ & $24.64 \pm 3.04$ & $4.82 \pm 0.66$ & $1.42 \pm 0.32$ \\
& Day 7 & $59.73 \pm 6.15$ & $4.72 \pm 0.49$ & $29.22 \pm 3.43$ & $2.58 \pm 0.77$ & $1.18 \pm 0.28$ \\
\hline \multirow{3}{*}{ Mild asphyxia group } & Day 1 & $64.38 \pm 4.43$ & $6.46 \pm 0.86$ & $24.23 \pm 3.32$ & $5.53 \pm 0.03$ & $1.39 \pm 0.20$ \\
& Day 3 & $61.23 \pm 3.32$ & $5.03 \pm 0.72$ & $27.12 \pm 2.88$ & $3.14 \pm 1.02$ & $1.22 \pm 0.15$ \\
& Day 7 & $59.08 \pm 3.02$ & $4.69 \pm 0.69$ & $30.88 \pm 3.01$ & $2.59 \pm 0.09$ & $1.11 \pm 0.09$ \\
\hline Control group & & $58.43 \pm 2.47$ & $4.51 \pm 0.87$ & $31.68 \pm 4.77$ & $2.44 \pm 0.77$ & $1.08 \pm 0.21$ \\
\hline
\end{tabular}

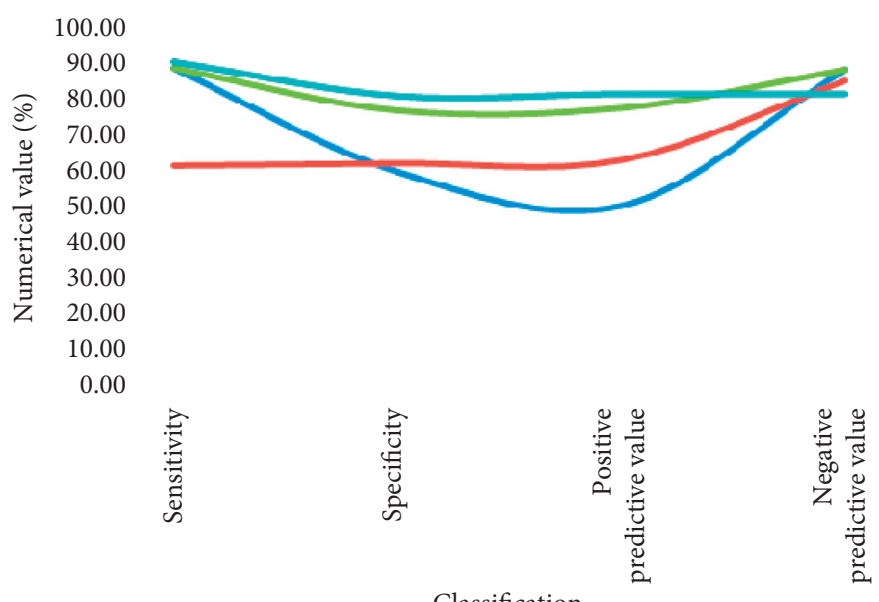

Classification

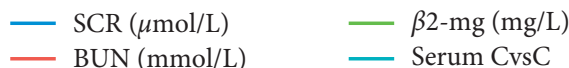

Figure 2: Diagnostic value.

TABLE 2: Diagnostic value.

\begin{tabular}{lcccc}
\hline Index & Sensitivity (\%) & Specificity (\%) & Positive predictive value (\%) & Negative predictive value (\%) \\
\hline SCR $(\mu \mathrm{mol} / \mathrm{L})$ & 88.47 & 59.49 & 50.00 & 88.09 \\
BUN $(\mathrm{mmol} / \mathrm{L})$ & 61.20 & 61.82 & 62.75 & 84.96 \\
$\beta 2-\mathrm{mg}(\mathrm{mg} / \mathrm{L})$ & 88.47 & 76.78 & 77.46 & 88.09 \\
Serum CysC & 90.07 & 80.66 & 81.25 & 81.25 \\
\hline
\end{tabular}




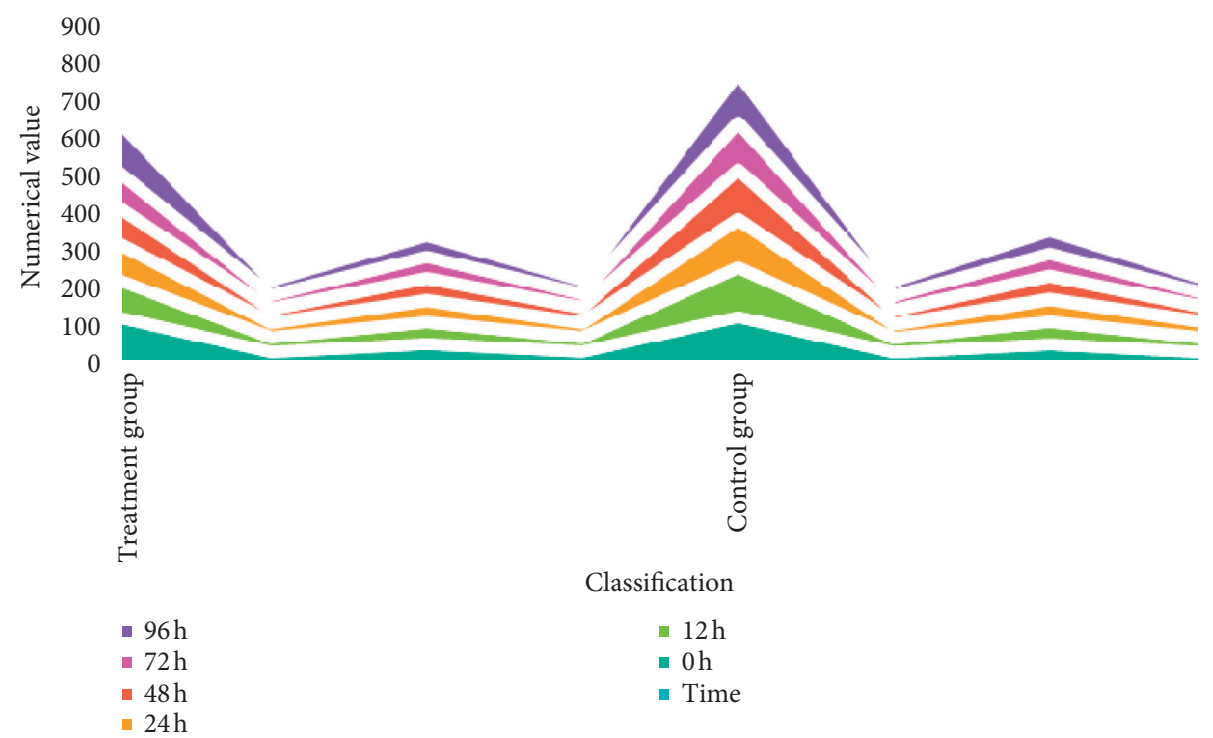

Figure 3: Changes in vital signs.

TABle 3: Changes in vital signs.

\begin{tabular}{|c|c|c|c|c|c|c|c|c|}
\hline \multirow{2}{*}{ Time } & \multicolumn{4}{|c|}{ Treatment group } & \multicolumn{4}{|c|}{ Control group } \\
\hline & Heart rate & Breathing & Systolic pressure & Diastolic pressure & Heart rate & Breathing & Systolic pressure & Diastolic pressure \\
\hline 0 & $131 \pm 8$ & $38 \pm 7$ & $59 \pm 10$ & $39 \pm 7$ & $130 \pm 9$ & $37 \pm 9$ & $58 \pm 9$ & $37 \pm 7$ \\
\hline 12 & $101 \pm 8$ & $39 \pm 7$ & $58 \pm 5$ & $38 \pm 3$ & $132 \pm 9$ & $38 \pm 8$ & $60 \pm 6$ & $38 \pm 7$ \\
\hline 24 & $97 \pm 13$ & $38 \pm 8$ & $57 \pm 5$ & $38 \pm 5$ & $127 \pm 7$ & $36 \pm 10$ & $59 \pm 7$ & $42 \pm 5$ \\
\hline 48 & $96 \pm 8$ & $35 \pm 12$ & $57 \pm 11$ & $39 \pm 6$ & $131 \pm 11$ & $36 \pm 9$ & $60 \pm 8$ & $38 \pm 8$ \\
\hline 72 & $97 \pm 5$ & $37 \pm 10$ & $58 \pm 5$ & $37 \pm 8$ & $127 \pm 10$ & $39 \pm 4$ & $60 \pm 5$ & $37 \pm 4$ \\
\hline 96 & $136 \pm 10$ & $39 \pm 4$ & $56 \pm 6$ & $36 \pm 3$ & $131 \pm 8$ & $39 \pm 6$ & $61 \pm 6$ & $38 \pm 5$ \\
\hline
\end{tabular}

temperature, but there was no significant difference with the control group and mild hypothermia treatment process.

4.2.2. Hepatorenal Function. According to the statistical analysis of data, as shown in Figure 4 and Table 4, ALT, AST, BUN, and SCR in the two groups were increased in different degrees before treatment. After symptomatic treatment, ALT, AST, BUN, and SCR of the two groups were improved in different degrees, which was statistically significant compared with that before treatment $(p<0.05)$. There was no significant difference between the two groups before and after treatment.

\section{Analysis and Discussion}

5.1. Analysis of Experimental Results. The results showed that the levels of serum $\beta 2$-mg and CysC were positively correlated, but negatively correlated with SCR, BUN, and GFR The decrease of glomerular filtration rate and the impairment of renal function can be explained from the following aspects: the change of renal artery hemodynamics: some scholars have observed the change of renal artery hemodynamics in nearly asphyxiated newborns through Doppler ultrasound and found that the decrease of diastolic blood supply may be the main cause of renal injury and

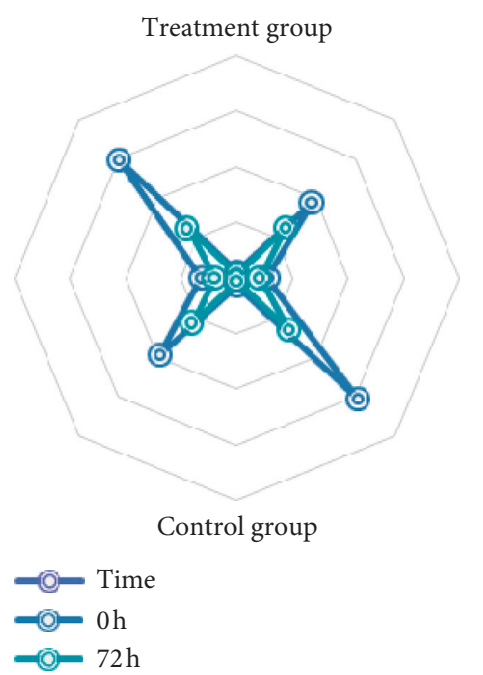

Figure 4: Hepatorenal function.

dysfunction after asphyxia. The systolic and diastolic phase of blood supply decreased, and the renal blood flow decreased. Blood flow mainly passes through the medulla of the kidney, resulting in short circuit of blood flow in the kidney and decrease of glomerular filtration rate. The important role of vasoactive substances in the occurrence and development 
TABLE 4: Hepatorenal function.

\begin{tabular}{lcccccccc}
\hline \multirow{2}{*}{ Time (h) } & \multicolumn{4}{c}{ Treatment group } & \multicolumn{3}{c}{ Control group } \\
& BUN & Cr & ALT & AST & BUN & Cr & ALT & AST \\
\hline 0 & $5.44 \pm 1.43$ & $95.40 \pm 39.87$ & $27.70 \pm 15.02$ & $154.15 \pm 94.70$ & $6.58 \pm 3.0$ & $98.34 \pm 46.64$ & $31.65 \pm 16.58$ & $149.70 \pm 93.06$ \\
72 & $3.01 \pm 2.02$ & $63.30 \pm 25.58$ & $20.57 \pm 10.67$ & $66.37 \pm 23.59$ & $3.07 \pm 1.44$ & $57.59 \pm 24.60$ & $19.59 \pm 6.15$ & $62.63 \pm 19.24$ \\
\hline
\end{tabular}

of multiple organ injury asphyxia: some scholars released clips, causing ischemia and reinjecting renal blood flow into renal cortex, and the glomerular filtration rate is still not increased, so it can be seen that simple renal blood perfusion decreased. All of these explain the phenomenon of glomerular filtration rate decrease. The activity of renin-a system increased significantly, resulting in vasospasm. The content of renin in the outer layer of renal cortex was the highest, so the renal cortex ischemia was the most serious. At the same time, the imbalance of prostaglandin system and balance can also lead to renal ischemia and decreased glomerular filtration rate. In addition, the increase of endothelin during asphyxia can reduce the glomerular ultrafiltration coefficient (KF) and glomerular filtration rate through the contraction of mesangial cells. Asphyxia and hypoxia can damage renal tissue, especially proximal renal tubular cells, leading to changes in cytoskeleton, cell polarity, and renal tubular blockage. However, the renal tubules were damaged after the reabsorption of sodium and chlorine decreased, and the tubules with increased concentration of sodium and chlorine reached the dense point and then made the entering artery contract, and the resistance increased. A vicious circle is formed, that is, the so-called tube ball feedback mechanism. The detection of serum cystatin $\mathrm{C}$ is helpful to understand the severity of neonatal asphyxia. Glomerular filtration has the following advantages: cystatin $\mathrm{C}$ is not easy to pass through the fetal mucus barrier. Foreign scholars have studied the serum cystatin $\mathrm{C}$ and creatinine levels of healthy pregnant women and newborns and found that there is a significant difference between the serum cystatin $\mathrm{C}$ levels of mothers and newborns, and there is no linear correlation between the two, so they think most of the cystatin $\mathrm{C}$ of newborns comes from themselves. Because of the lobulation of the new kidney, the small glomerulus, the small filtering area of the membrane, and the short tubules of the new kidney, especially the proximal tubules, are only $1 / 10$ of those of the adult.

The metabolic pathway of creatinine in newborns is relatively complex. In addition to filtering the renal ice, the renal tubules also discharge part of the renal ice out of the body. The excretion of urea nitrogen in the kidney and the concentration in the blood are significantly affected by the water content and urine volume of the human body. In preterm infants, increased renal reabsorption of creatinine affects serum creatinine concentration, whereas cystatin $\mathrm{C}$ does not. Once filtered, they are usually absorbed and broken down by epithelial cells near the convoluted tubules. Cystatin $\mathrm{C}$ is suitable for monitoring renal function due to its physical and chemical properties. Neonatal jaundice and hemolysis will affect the determination of creatinine levels through bilirubin, hemoglobin, and other factors. In addition, the serum cystatin $\mathrm{C}$ concentration is very stable and can be maintained at room temperature for two days. The biological characteristics of cystatin $\mathrm{C}$ also make it a marker of glomerular filtration. Generally, we use the determination of material clearance rate as the most convenient method to evaluate the ability of kidney to control the concentration of extracellular fluid. Inulin and its isotopic labeled complex limit its routine clinical application, so some endogenous markers such as creatinine, urea, and uridine are often used to determine or selectively directly determine the plasma concentration (or their interaction). The latter are urea nitrogen, creatinine, and microglobulin. However, exogenous and endogenous markers are not stable in the blood; some of them are affected by other pathological changes, muscle mass, and diet.

Cystatin $\mathrm{C}$ is a direct gene product in recent years, which is a small molecule protein. Its synthesis is not affected by muscle mass and acute inflammatory response. Its tissue formation rate is quite stable; almost all of them are filtered by glomeruli, absorbed, decomposed, and metabolized in proximal convoluted tubules. It has many advantages in the evaluation of glomerular filtration function and has been paid more and more attention. It can also inhibit papain, fig protease, and cathepsins $\mathrm{H}$ and L. Therefore, the physiological function of cystatin $\mathrm{C}$ may be to regulate the activity of caspase. The mutation of caspase inhibitor $\mathrm{C}$ gene will lead to the rupture of cerebral artery and caspase inhibitor $\mathrm{C}$ (HCCAA) in hereditary amyloidosis. This is a clinical disease directly related to caspase inhibitor $C$, because caspase inhibitor $\mathrm{C}$ can be specifically expressed in almost all cell tissues. The rate of formation of caspase inhibitor $C$ in the body is quite stable. In pediatric patients, neonatal jaundice and hemolysis will be interfered by bilirubin, hemoglobin, and other factors, which will affect the determination of creatinine level in the blood.

5.2. Discussion. The most common complication of neonatal asphyxia is dysfunction of brain, kidney, heart, and other organs, and kidney is one of the most vulnerable target organs. The incidence of severe asphyxia was high, because the blood flow of human kidney is very large, accounting for about $20 \%$ of cardiac output. The renal blood flow of newborn is small, accounting for only about $8 \% \sim 12 \%$ of cardiac output in one week. After asphyxia, it is emphasized that the body's blood redistribution, the excessive accumulation of nitric oxide (no) in the local kidney, and the enhanced synthesis and the expression of intercellular adhesion molecules will damage the renal tissue; because the renal medulla is short, the transport of sodium, chlorine, and urinary hormones is weak, the permeability gradient of skin 
and renal medulla is low, and the response of distal nephron to diuretic hormones is low. Prostaglandin interferes with the concentration mechanism. It affects the concentration function of kidney, especially in the case of insufficient blood perfusion and hypoxia. Therefore, when neonatal asphyxia occurs, renal blood vessels contract and blood perfusion decreases.

At the same time, the serum BUN and Cr are affected by many factors, such as blood volume, protein intake, and so on. Mild hypothermia can reduce energy metabolism, inhibit brain cell apoptosis, reduce the release of excitatory amino acids at the end of axon, inhibit nitric oxide synthetase, and significantly improve prognosis. When the body temperature drops, the blood viscosity may rise, the curve of oxygen dissociation may shift to the left, the utilization of oxygen by tissues may be reduced, causing metabolic acidosis, bradycardia, arrhythmia, the decrease of cellular immune function, the increase of infection risk, the prolongation of coagulation time, the abnormality of platelet function, and the slight drop of blood potassium, chorea, etc. The core body temperature causes water to build up in the respiratory tract and medium, which can lead to slow breathing. If the temperature is too low, the breathing stops, but a slight malaise and shortness of breath can affect breathing. Excessive cooling can cause brain damage by the following actions: polymorphonuclear leukocytes attach to the walls of blood vessels to exacerbate cerebral ischemia. Negative reactions released by the vaccine. Small blood vessels can cause a technological breakdown and increase the expression of some genes after ischemia.

Effects of hypothermia on IEO expression: IEQ is a group of genes that express rapidly and transiently after cerebral ischemia. Transient hemicerebral ischemia can induce a large amount of immediate early gene expression. Mild hypothermia promoted the late stage of gene expression, which was the result of rapid activation of cytoplasmic response elements after ischemia injury. The direct induction of early genes is a process of activation and repair of stress signals. In the early stage, the induction accelerating gene of mild hypothermia for the treatment of cerebral ischemia reflects the rapid recovery of signal transduction function to normal cells, so as to improve the repair ability of neurons. The effect of low temperature on HSP gene expression: HSP is a group of stress-induced protein HSP genes in biological cells after activation of some environmental factors and stress stimulation. At present, many kinds of heat shock proteins have been found, among which the most conservative and important is the Hsp70 family. Hsp70 family mainly includes HSP68, 72, 73, HSC (heat shock homology) 70, and hsp78. Among them, HSP70 and HSP72 are the most studied in depth in ischemic injury. HSP72 protein expression is a useful marker of stress response after cerebral ischemia. Mild hypothermia is safe and feasible for newborns. Although the current clinical trials show that mild hypothermia has no obvious side effects in a short period of time, it is undeniable that mild hypothermia can affect the whole body organ system. Understanding the pathophysiological changes of mild hypothermia is of great significance for the safe implementation of mild hypothermia treatment. In clinical treatment, we should pay attention to the side effects of any treatment measures. The side effects of perinatal asphyxia and hypothermia are obviously overlapped, which brings some difficulties to clinical observation. We should be more careful. Hypothermia has a certain impact on all organs and systems of the whole body: during the treatment of hypothermia, the change and rewarming of cardiovascular system were observed, and the increase of mean arterial pressure was found. It causes the increase of vascular resistance, high viscosity and blood concentration, thrombocytopenia, and coagulation abnormality. In addition, due to the influence of platelet function, prothrombin, and partial thrombin time, the bleeding time is prolonged, which can cause circulatory failure and DIC. Hypothermia can lead to immunosuppression, inhibit leukocyte migration and phagocytosis, and easily increase septicemia, especially pneumonia. The level of antidiuretic hormone decreased. Low temperature can increase metabolic rate, increase oxygen consumption, shift oxygen separation curve to the left, decrease drug metabolism, hypoglycemia, metabolic acid, and low potassium, increase catecholamine, and stimulate glycogen release to cause hyperglycemia, etc. Cortisol increased, TSH increased, and antidiuretic hormone decreased. In the process of rewarming, as a result of vasodilation and decreased blood flow to the heart and the decrease of effective circulation blood volume and blood pressure, it leads to hypovolemic shock. When reheating, the intracranial pressure will suddenly rise. Low temperature rebound causes high temperature. When rewarming, the release of glycogen suddenly decreased, resulting in hypoglycemia, etc.

\section{Conclusion}

Serum CysC level was positively correlated with SCR and BUN and negatively correlated with GFR. The sensitivity and specificity of serum $\mathrm{CysC}$ in the diagnosis of renal function damage were higher than other indicators, which could be used as early indicators for monitoring renal damage in asphyxiated neonates. Combined with the biological and physicochemical characteristics of cystatin $\mathrm{C}$, we believe that cystatin $\mathrm{C}$ is an ideal endogenous marker to reflect the early renal function damage in neonates. Mild hypothermia treatment has better therapeutic effect.

\section{Data Availability}

No data were used to support this study.

\section{Conflicts of Interest}

The authors declare that there are no conflicts of interest in this paper.

\section{References}

[1] H. Kletkiewicz, A. Nowakowska, A. Siejka et al., "Deferoxamine prevents cerebral glutathione and vitamin E depletions in asphyxiated neonatal rats: role of body temperature," 
International Journal of Hyperthermia, vol. 32, no. 2, pp. 211-220, 2016.

[2] M.-H. Gagnon and P. Wintermark, "Effect of persistent pulmonary hypertension on brain oxygenation in asphyxiated term newborns treated with hypothermia," The Journal of Maternal-Fetal \& Neonatal Medicine, vol. 29, no. 13, pp. 2049-2055, 2016.

[3] A. A. Balushi, S. B. Vargas, J. Maluorni et al., "Hypotension and brain injury in asphyxiated newborns treated with hypothermia," American Journal of Perinatology, vol. 35, no. 01, pp. 031-038, 2018.

[4] A. Yazdani, Z. Khoja, A. Johnstone, L. Dale, E. Rampakakis, and P. Wintermark, "Sildenafil improves brain injury recovery following term neonatal hypoxia-ischemia in male rat pups," Developmental Neuroscience, vol. 38, no. 4, pp. 251-263, 2016.

[5] I. Oloyede and P. Udo, "An audit of some health facilities and equipment for neonatal resuscitation in south-south Nigeria," Nigerian Journal of Paediatrics, vol. 43, no. 3, p. 197, 2016.

[6] L. Renter Valdovinos and I. Oulego Erroz, "Ecografía a pie de cama en el niño crítico," Anales de Pediatría, vol. 85, no. 3, pp. 117-118, 2016.

[7] T. Niranjan, A. Thangaraj, B. Vishnu et al., "Phase changing material for therapeutic hypothermia in neonates with hypoxic ischemic encephalopathy-a multi-centric study," Indian Pediatrics, vol. 55, no. 3, pp. 201-205, 2018.

[8] D. E. Clay, A. C. Linke, D. J. Cameron et al., "Evaluating affordable cranial ultrasonography in east african neonatal intensive care units," Ultrasound in Medicine \& Biology, vol. 43, no. 1, pp. 119-128, 2017.

[9] C. Jin, M. Feng, J. Zhong et al., "Insights into mechanism of size controlled synthesis of $\mathrm{CH} 3 \mathrm{NH} 3 \mathrm{PbBr} 3$ perovskite quantum dots and large nanoparticles with tunable optical properties," Organic Electronics, vol. 82, Article ID 105172, 2020.

[10] D. K. Mandal, P. Kumar, U. S. Prasad, S. Datta, I. Dawn, and S. Sarkar, "Etiological factors \& clinical courses of birth asphyxia in rural and urban population of Kishanganj district," Bangladesh Journal of Medical Science, vol. 16, no. 4, pp. 554-556, 2017.

[11] L. Vega, H. Boix, D. Albert, I. Delgado, and F. Castillo, "El intervalo QT corregido durante la hipotermia terapéutica en la encefalopatía hipóxico-isquémica," Anales de Pediatría, vol. 85, no. 6, pp. 312-317, 2016.

[12] Y. J. Xu, L. M. Ran, S. S. Zhai et al., "Evaluation of the efficacy of atosiban in pregnant women with threatened preterm labor associated with assisted reproductive technology," European Review for Medical and Pharmacological Sciences, vol. 20, no. 9, p. 1881, 2016.

[13] T. Yasmin, S. Akther, S. Sultana, and M. B. Amin, "Assessment of cranial sonographic findings of hypoxic ischemic brain injury in perinatal asphyxia," Journal of Medicine, vol. 17, no. 1, p. 12, 2016.

[14] J. Mori, C. Tanikawa, Y. Funauchi, P. H. Y. Lo, Y. Nakamura, and K. Matsuda, "Cystatin C as a p53-inducible apoptotic mediator that regulates cathepsin L activity," Cancer Science, vol. 107, no. 3, pp. 298-306, 2016.

[15] M. Schanz, D. Pannes, J. Dippon, C. Wasser, M. D. Alscher, and M. Kimmel, "The influence of thyroid function, inflammation, and obesity on risk prediction of acute kidney injury by cystatin C in the emergency department," Kidney and Blood Pressure Research, vol. 41, no. 5, pp. 604-613, 2016.

[16] K. K. Lee, H. C. Tseng, Y. M. Hwu et al., "Expression of cystatin $\mathrm{C}$ in the female reproductive tract and its effect on human sperm capacitation," Reproductive Biology and Endocrinology, vol. 16, no. 1, p. 8, 2018.

[17] M. Bi, Z. Huang, P. Li, C. Cheng, and andW. Chen, "The association between elevated cystatin $\mathrm{C}$ levels with myocardial infarction: a meta-analysis," International Journal of Clinical and Experimental Medicine, vol. 8, no. 11, pp. 20540-20547, 2016.

[18] J. Zhao, W. Deng, Y. Zhang et al., "Association between serum cystatin $\mathrm{C}$ and diabetic foot ulceration in patients with type 2 diabetes: a cross-sectional study," Journal of Diabetes Research, vol. 2016, no. 6, pp. 1-7, Article ID 8029340, 2016.

[19] A.-S. Bargnoux, L. Piéroni, J.-P. Cristol et al., "Multicenter evaluation of cystatin $\mathrm{C}$ measurement after assay standardization," Clinical Chemistry, vol. 63, no. 4, pp. 833-841, 2017.

[20] S. Lemoine, M. Panaye, C. Pelletier et al., "Cystatin C-creatinine based glomerular filtration rate equation in obese chronic kidney disease patients: impact of deindexation and gender," American Journal of Nephrology, vol. 44, no. 1, pp. 63-70, 2016.

[21] S.-K. Yang, J. Liu, X.-M. Zhang et al., "Diagnostic accuracy of serum cystatin $\mathrm{C}$ for the evaluation of renal dysfunction in diabetic patients: a meta-analysis," Therapeutic Apheresis and Dialysis, vol. 20, no. 6, pp. 579-587, 2016.

[22] A. Barbati, B. Cappuccini, M. C. Aisa et al., "Increased urinary cystatin-C levels correlate with reduced renal volumes in neonates with intrauterine growth restriction," Neonatology, vol. 109, no. 2, pp. 154-160, 2016. 